\title{
SCHUR MULTIPLIERS OF SOME FINITE NILPOTENT GROUPS
}

\author{
DAVID A. JACKSON
}

\begin{abstract}
Let $B$ denote the Burnside group, $B\left(p^{\alpha}, d\right)$ and let $G=B / B_{k}$ where $p$ is a prime and $1<k<p$. We show that the Schur multiplier, $M(G)$, is a direct power of $\Psi(k, d)$ cyclic groups, each having order $p^{\alpha}$, where $\Psi(k, d)=k^{-1} \Sigma_{n \mid k} \mu(k / n) d^{n}$. (This is Witt's formula for the rank of $F_{k} / F_{k+1}$ where $F$ is free on $d$ generators.) In addition we can show that $\boldsymbol{M}(B(3, d))$ is elementary abelian of exponent 3 and rank $2\left(\frac{d}{2}\right)+4\left(\frac{d}{3}\right)+$ $3\left(\begin{array}{c}d \\ 4\end{array}\right)$.
\end{abstract}

1. Notation and a preliminary result. For any group $G, M(G)$ will be the Schur multiplier of $G$. The minimal number of generators for $G$ will be denoted $d(G)$. When $G$ is abelian we will sometimes refer to $d(G)$ as the rank of $G$ and write $\operatorname{rk}(G) . Z(G)$ will be the center of $G$ and $G^{\prime}$ will be the commutator subgroup of $G$. $G_{k}$ will be the $k$ th term of the lower central series $G_{1}=G, G_{2}=G^{\prime}=(G, G), G_{3}=\left(G_{2}, G\right), \ldots$

Throughout this paper $d$ will be a fixed positive integer, $p$ will be a fixed prime and $q$ will be $p^{\alpha}$ for some fixed positive integer $\alpha$. $F$ will be a free group on a set $\left\{x_{1}, x_{2}, \ldots, x_{d}\right\}$ of $d$ generators, and $\Psi(k, d)$, the rank of $F_{k} / F_{k+1}$, will be abbreviated $\Psi(k)$. $B(q, d)$ will be the Burnside group with exponent $q$ on $d$ generators.

I thank N. Blackburn for showing me a proof of the following generalization of a theorem of R. C. Lyndon [7].

TheORem 1.1. Let $B=B(q, d)$. Then $B_{k} / B_{k+1}$ is a direct sum of $\Psi(k)$ cyclic groups of order $q$, for $k<p$.

Proof. We claim that $F_{k} \cap F^{q} F_{k+1}=F_{k}^{q} F_{k+1}$ for $k<p$, hence

$$
B_{k} / B_{k+1} \cong F^{q} F_{k} / F^{q} F_{k+1} \cong F_{k} / F_{k} \cap F^{q} F_{k+1} \cong F_{k} / F_{k}^{q} F_{k+1} \text {. }
$$

To prove the claim we show by induction on $j$, for $1 \leqslant j \leqslant k$, that $F_{j} \cap F^{q} F_{k+1} \subseteq F_{j}^{q} F_{k+1}$. Given $w \in F_{j} \cap F^{q} F_{k+1}$, then $w \in F_{j-1} \cap F^{q} F_{k+1}$, so by induction $w \in F_{j-1}^{q} F_{k+1}$. After moving the factors from $F_{k+1}$ to the right, we can write $w=a_{1}^{q} \cdots a_{r}^{q} x$ where $a_{i} \in F_{j-1}$ and $x \in F_{k+1}$. Now consider $\left(a_{1} a_{2} \cdots a_{r}\right)^{q}$ and apply Theorem 12.3.1 of [2] to get

$$
\left(a_{1} a_{2} \cdots a_{r}\right)^{q}=a_{1}^{q} a_{2}^{q} \cdots a_{r}^{q} c_{1}^{e_{1}} \cdots c_{t}^{e^{r} y}
$$

Received by the editors September 22, 1976.

AMS (MOS) subject classifications (1970). Primary 20C25; Secondary $18 \mathrm{H} 10$.

Key words and phrases. Schur multiplier, Burnside group, commutator calculus. 
where $y \in F_{k+1}$, each $c_{i} \in F_{j}$ and $q$ divides each $e_{i}$ since $k<p$. Observe that $w x^{-1}=a_{1}^{q} \cdots a_{r}^{q} \in F_{j}$, so $\left(a_{1} a_{2} \cdots a_{r}\right)^{q} \in F_{j}$. Since $F / F_{j}$ is torsion free, $a_{1} a_{2} \cdots a_{r} \in F_{j}$. But then since $q$ divides each $l_{i}$, we get $a_{1}^{q} \cdots a_{r}^{q} \in$ $F_{j}^{q} F_{k+1}$.

\section{The exponent of $M(G)$.}

TheOREM 2.1. Let $B=B(q, d), G=B / B_{k}$ where $k<2 p$ and $Q=G / N$ where $N \subseteq G_{l}$ and $l>k-p$. Then $M(Q)$ has exponent dividing $q$.

Proof. We may assume that $d$ is chosen so that $d(Q)=d(G)=d$ and that $k$ is chosen so that class $Q=$ class $G=k-1$.

Consider an exact sequence $M \mapsto H \rightarrow \rightarrow^{\pi} Q$ where $M \subseteq H^{\prime} \cap Z(H)$. Then $H$ is a finite $p$-group with $d(H)=d$ and class $H \leqslant k$. Let $\left\{z_{1}, z_{2}, \ldots, z_{d}\right\}$ be a set of generators for $Q$ and let $\left\{h_{1}, h_{2}, \ldots, h_{d}\right\}$ be a set of generators for $H$ with $\pi h_{i}=z_{i}$. For any commutator $\bar{c}$ in $Q$ with entries from $\left\{z_{i}\right\}$ we will let $c$ denote the corresponding commutator in $H$ with each $z_{i}$ replaced by $h_{i}$.

Observe that for any $h_{i}, h_{i}^{q} \in M$, and thus is central. Hence given $c=$ $\left(h_{i}, \ldots, h_{i j}\right)$, we have $\left(c H_{j+1}\right)^{q}=\left(h_{i}, \ldots, h_{i_{j}}^{q}\right) H_{j+1}=H_{j+1}$. It follows that $H_{j} / H_{j+1}$ has exponent dividing $q$. We wish to show that $H_{k-p+1}$ has exponent dividing $q$. We show by induction on $\lambda$, for $0 \leqslant \lambda<p$, that $H_{k-\lambda}$ has exponent dividing $q$. Given $\lambda<p$, write $r=k-\lambda$, so that $r+p>k$. Then for any commutator $c=\left(h_{i}, \ldots, h_{i}\right) \in H_{r}$ we use, e.g., Lemma $\mathrm{H} 2$ of [9] to write

$$
1=\left(h_{i_{1}}, \ldots, h_{i_{r}}^{q}\right)=c^{q} c_{1}^{e_{1}} \cdots c_{t}^{e_{t}}
$$

where the $c_{i}$ are all commutators with $r<w t c_{i} \leqslant k$. Since $p+r>k, q$ divides each $e_{i}$. By induction the $c_{i}$ have exponent dividing $q$, so $c$ has exponent dividing $q$ as well. For an arbitrary element $x$ of $H_{k-\lambda}$, we may write $x$ as a product of commutators of weight at least $k-\lambda$ and apply Theorem 12.3.1 of [2] together with the above result for commutators to show that $x$ has exponent dividing $q$.

Thus to prove the theorem it suffices to show that $M \subseteq H_{k-p+1}$. Since $l \geqslant k-p+1$ and $p \geqslant k-p+1$ we are done if we show either that $M \subseteq H_{l}$ or that $M \subseteq H_{p}$. We find the remainder of the argument easier to follow if we separate the cases $l<p$ and $l \geqslant p$.

Case (i) $l<p$. We show in this case that $M \subseteq H_{l}$. For each $j$, choose a basis $\left\{\bar{c}_{1} Q_{j+1}, \ldots, \bar{c}_{r(j)} Q_{j+1}\right\}$ for $Q_{j} / Q_{j+1}$, taking $\left\{z_{1} Q_{2}, z_{2} Q_{2}, \ldots, z_{d} Q_{2}\right\}$ as the choice for $Q / Q_{2}$. Fix a weight preserving order of all of the $\bar{c}$ and relabel according to this order, so that (up to the choice of bases and the choice of the order) each element $w$ of $Q$ has a unique expression $w=\prod \bar{c}_{i}^{\bar{\alpha}_{i}}$ where $\bar{c}_{i} \in Q_{j}, \bar{c}_{i} Q_{j+1}$ has order $\bar{\beta}_{i}$ in $Q_{j} / Q_{j+1}$ and $0 \leqslant \bar{\alpha}_{i}<\bar{\beta}_{i}$.

For $j<l, Q_{j} / Q_{j+1} \cong B_{j} / B_{j+1}$, hence $r(j)=\Psi(j)$ and $Q_{j} / Q_{j+1}$ is homocyclic of exponent $q$. We have seen above that $H_{j} / H_{j+1}$ has exponent dividing $q$. Since $Q_{j} / Q_{j+1}$ is an image of $H_{j} / H_{j+1}$, the latter must have rank $\Psi(j)$ and exponent $q$ for $j<l$. The corresponding sets 
$\left\{c_{1} H_{j+1}, \ldots, c_{\Psi(j)} H_{j+1}\right\}$ form bases for $H_{j} / H_{j+1}$ when $j<l$. Choose, as well, bases for $H_{j} / H_{j+1}$ when $j \geqslant l$. Order those $c_{i}$ with weight less than $l$ in the same way as the $\bar{c}_{i}$ were ordered, and extend this order to a weight preserving order of all of the $c_{i}$ 's. Then each element $h$ of $H$ has a unique expression $h=\Pi c_{i}^{\alpha_{i}}$ where $c_{i} \in H_{j}, c_{i} H_{j+1}$ has order $\beta_{i}$ in $H_{j} / H_{j+1}$ and $0 \leqslant \alpha_{i}<\beta_{i}$. If we apply the quotient map $\pi$ to $h$, we get

$$
\pi h=\pi \Pi c_{i}^{\alpha_{i}}=\prod \pi c_{i}^{\alpha_{i}}=\prod_{w t c_{i}<l} \bar{c}_{i}^{\alpha_{i}} \cdot \prod_{w t c_{i}>l} \bar{c}_{i}^{\alpha_{i}^{\prime}}
$$

so that the unique expression for $\pi h$ has $\bar{\alpha}_{i}=\alpha_{i}$ for $w t c_{i}<l$ and $\bar{\alpha}_{i}$ equal to some $\alpha_{i}^{\prime}$ when $w t c_{i} \geqslant l$. If $h \in M$, then $\pi h=1$, so $\bar{\alpha}_{i}=0$ for all $i$. Hence $\alpha_{i}=0$ for $w t c_{i}<l$ and $h \in H_{l}$.

Case (ii) $l \geqslant p$. The argument here is the same as in Case (i) except we observe that $Q_{j} / Q_{j+1} \cong B_{j} / B_{j+1} \cong H_{j} / H_{j+1}$ for $j<p$ and show that $M \subseteq$ $H_{p}$.

Corollary 2.2. Let $B=B(q, d), G=B / B_{k}$ where $p \leqslant k<2 p$ and let $M \rightarrow H \rightarrow G$ be exact with $M \subseteq H^{\prime} \cap Z(H)$. Then $M \subseteq H_{p}$.

Corollary 2.3. Let $B=B(q, d)$ and $G=B / B_{k}$ where $k<2 p$. Then $M(G)$ has exponent dividing $q$.

COROLlaRY 2.4. If $Q$ is any group of exponent 3, then $M(Q)$ has exponent dividing 3.

Proof. Let $d=d(Q)$ and $B=B(3, d)$. Then $B=B / B_{4}$ (see [2]) and $Q=B / N$ for some $N \subseteq B_{2}$.

Corollary 2.4 is implicit in Remark 2.8 of Jones [5] since any group of exponent 3 satisfies the 2nd Engel condition.

We do not have any example to show that the condition on $k$ in Theorem 2.1 is necessary. The example of Bayes, Kautsky, and Wamsley [1] of a group $G$ with exponent 4 with $M(G)$ having exponent 8 shows that the hypothesis on $l$ cannot be dropped.

\section{The multiplier of groups having exponent $q$.}

THEOREM 3.1. If $B=B(q, d)$ and $G=B / B_{k}$ where $1<k<p$, then $M(G)$ is homocyclic with exponent $q$ and rank $\Psi(k)$.

Proof. Observe first that $B_{k} / B_{k+1} \mapsto B / B_{k+1} \rightarrow G$ is exact with $B_{k} / B_{k+1}$ in both the commutator and central subgroups of $B / B_{k+1}$. It follows from a theorem of Schur, see [3, V, Satz 23.5], that $B_{k} / B_{k+1}$ is an epimorphic image of $M(G)$. Then by Theorem 1.1 we see that rk $M(G) \geqslant \Psi(k)$ and $|M(G)| \geqslant$ $q^{\Psi(k)}$.

By Corollary 2.3, $M(G)$ has exponent dividing $q$, so it will suffice to show that rk $M(G) \leqslant \Psi(k)$. To this end we exhibit a presentation for $G$.

Let $\left\{c_{1} F_{k+1}, \ldots, c_{\Psi(k)} F_{k+1}\right\}$ be a basis for $F_{k} / F_{k+1}$. Then we claim that $G$ has a presentation 


$$
\left\langle x_{1}, x_{2}, \ldots, x_{d} \mid x_{1}^{q}, x_{2}^{q}, \ldots, x_{d}^{q}, c_{1}, \ldots, c_{\Psi(k)}, F_{k+1}\right\rangle .
$$

Let $\bar{G}$ be the group having this presentation. The obvious function $\bar{G} \rightarrow G$ preserves relations, and hence is a group epimorphism. $\bar{G}$ is nilpotent of class $k-1$ and rk $\bar{G}_{j} / \bar{G}_{j+1} \leqslant \Psi(j)=\mathrm{rk} G_{j} / G_{j+1}$. We argue as in Theorem 2.1 that each $\bar{G}_{j} / \bar{G}_{j+1}$ has exponent dividing $q$. It follows that $\left|\bar{G}_{j} / \bar{G}_{j+1}\right| \leqslant$ $\left|G_{j} / G_{j+1}\right|$ so that $|\bar{G}| \leqslant|G|$.

Having this presentation for $G$, it follows, as in [3, V, Satz 25.2], that rk $M(G) \leqslant \Psi(k)$. Since $G$ is nilpotent of class $k-1$, the relators in $F_{k+1}$ contribute nothing to the number of generators of $M(G)$.

It would be of interest to know if $F_{k+1}$ can be omitted from the set of relators in the above presentation. If so, the resulting presentation has deficiency $-\mathrm{rk} M(G)$.

Proposition 3.2. If $B=B(q, d)$ and $G=B / B_{p}$ then $M(G)$ has exponent dividing $q$ and $\mathrm{rk} M(G) \leqslant \Psi(p)$.

Proof. Let $M \mapsto H \rightarrow G$ be exact with $M \subseteq H^{\prime} \cap Z(H)$. By Corollary 2.2, $M \subset H_{p}$. But $H_{p+1}=1$, so rk $H_{p} \leqslant \Psi(p)$.

Using results of Lyndon [7], one can easily show that

$$
\operatorname{rk} M(G) \geqslant \Psi(p)+d-\left(\begin{array}{c}
p+d-1 \\
p
\end{array}\right) \text { for } G=B / B_{p}
$$

The difficulty in improving the result here is that we do not have a convenient representation group to exhibit.

4. The multipliers of groups of exponent 3. Throughout this section we let $B=B(3, d) . B$ has been described by Levi and van der Waerden [6]. For details see, for example, [2]. Our present proofs of results in this section are tedious, but straightforward, and we condense or omit them.

LEMMA 4.1. B has presentations with $d$ generators and $r=d+2\left(\begin{array}{l}d \\ 2\end{array}\right)+4\left(\begin{array}{l}d \\ 3\end{array}\right)+$ $3\left(\begin{array}{l}d \\ 4\end{array}\right)$ relators.

Proof. One such presentation is

$$
\begin{aligned}
\left\langle x_{1}, x_{2}, \ldots, x_{d}\right| x_{i}^{3} & =1,1 \leqslant i \leqslant d, \\
\left(x_{j}, x_{i}, x_{i}\right) & =\left(x_{j}, x_{i}, x_{j}\right)=1,1 \leqslant i<j \leqslant d, \\
\left(x_{k}, x_{j}, x_{i}, x_{i}\right) & =\left(x_{k}, x_{j}, x_{i}, x_{j}\right)=\left(x_{k}, x_{j}, x_{i}, x_{k}\right) \\
& =\left(x_{k}, x_{j}, x_{i}\right)\left(x_{k}, x_{i}, x_{j}\right)=1,1 \leqslant i<j<k \leqslant d, \\
\left(x_{l}, x_{k}, x_{j}, x_{i}\right) & =\left(x_{l}, x_{k}, x_{i}, x_{j}\right)=\left(x_{l}, x_{j}, x_{i}, x_{k}\right)=1, \\
& 1 \leqslant i<j<k<l \leqslant d\rangle .
\end{aligned}
$$

LEMMA 4.2. There is a group $H$ and a short exact sequence $M \mapsto H \rightarrow B$ where $M \subseteq Z(H) \cap H^{\prime}$ and $M$ is elementary abelian of exponent 3 and rank $r-d=2\left(\begin{array}{l}d \\ 2\end{array}\right)+4\left(\begin{array}{l}d \\ 3\end{array}\right)+3\left(\begin{array}{c}d \\ 4\end{array}\right)$.

The presentation in Lemma 4.1 can be regarded as a blueprint for 
constructing such a representation group $H$. The details are tedious to verify.

THEOREM 4.3. $M(B)$ is elementary abelian of exponent 3 and rank $r-d=$ $2\left(\begin{array}{l}d \\ 2\end{array}\right)+4\left(\begin{array}{c}d \\ 3\end{array}\right)+3\left(\begin{array}{c}d \\ 4\end{array}\right)$.

Proof. This follows easily from Lemmas 4.1 and 4.2.

We remark that MacDonald [8] has previously computed the rank of $M(B(3,3))$ as an application of a computer algorithm.

For the case $q=3$, we can improve on Proposition 3.2.

Proposition 4.2. Let $G=B / B_{3}$. Then $M(G)$ has exponent 3 and rank $\Psi(3)$.

Representation groups for $G=B / B_{3}$ are much easier to construct than those for $B$. We note, however, that, with a proper choice $H$ of a representation group for $\mathrm{B}, \mathrm{H} / \mathrm{H}_{4}$ is a representation group for $G$.

As a final remark, we note that essentially all of the multipliers that we are obtaining are nontrivial and that this is not surprising in view of $D$. $L$. Johnson's recent paper [4].

\section{REFERENCES}

1. A. J. Bayes, J. Kautsky and J. W. Wamsley, Computation in nilpotent groups (application), Proc. Second Internat. Conf. Theory of Groups, Lecture Notes in Math., vol. 372, SpringerVerlag, Berlin and New York, 1974. MR 50 \# 7299.

2. Marshall Hall, Jr., Theory of groups, Macmillan, New York, 1959.

3. B. Huppert, Endliche Gruppen. I, Springer-Verlag, Berlin and New York, 1967.

4. David L. Johnson, A property of finite p-groups with trivial multiplicator, Amer. J. Math. 98 (1976), 105-108. MR 53 \#603.

5. M. R. Jones, Some inequalities for $M(G)$. II, Proc. Amer. Math. Soc. 45 (1974), 167-172. MR 50 \#4741.

6. F. Levi and B. L. van der Waerden, Über eine besondere Klasse von Gruppen, Abh. Math. Sem. Univ. Hamburg 9 (1933), 154-158.

7. R. C. Lyndon, On Burnside's problem, Trans. Amer. Math. Soc. 77 (1954), 202-215. MR 16, 218.

8. I. D. MacDonald, A computer application to finite p-groups, J. Austral. Math. Soc. 17 (1974), 102-112.

9. Ruth Rebekka Struik, On nilpotent products of cyclic groups, Canad. J. Math. 12 (1960), 447-462. MR 22 \#11028.

Department of Mathematics, University of Illinois at Urbana-Champaign, Urbana, ILLINOIS 61801

Current address: Department of Mathematics, Oklahoma State University, Stillwater, Oklahoma 74074 